\title{
THE FIXED-POINT INDEX AND THE FIXED-POINT THEOREMS OF 1-SET-CONTRACTION MAPPINGS
}

\author{
LI GUOZHEN
}

(Communicated by John B. Conway)

\begin{abstract}
W. V. Petryshyn [1] studied the fixed-point theorem of 1-setcontraction mappings. This paper gives the definition of the fixed-point index of 1-set-contraction mappings and the concept of semiclosed 1-set-contraction mappings; then we obtain some fixed-point theorems about it.
\end{abstract}

In this paper we define the fixed-point index of 1-set-contraction mappings and give the concept of semiclosed 1-set-contraction mappings. Hence the fixedpoint index of strict-set-contraction mappings (Nussbaum [2]) is extended to 1-setcontraction mappings. We obtain furthermore some fixed-point theorems of semiclosed 1-set-contraction mappings. Let $E$ be a real Banach space, $X$ a nonempty closed convex subset of $E, D$ a relative bounded open set with respect to $X$, and $\bar{D}$ and $\partial D$ the closure and boundary of $D$ in $X$ respectively.

Suppose that $T: \bar{D} \rightarrow X$ is 1-set-contraction mapping and $\theta \notin \overline{(I-T) \partial D}$, so there exists $\delta>0$ such that

$$
\inf _{x \in \partial D}\|x-T x\| \geq \delta .
$$

We set $T_{k}=k T$, where $k \in(1-\delta / M, 1), M=\sup _{x \in \bar{D}}\|T x\|+\delta$. Obviously $T_{k}$ is a strict-set-contraction mapping. By Amann [3], $T_{k}$ has fixed-point index $i\left(T_{k}, D\right)$.

DEFinition 1. The fixed-point index $i(T, D)$ of $T$ on $D$ is defined by $i(T, D)=$ $i\left(T_{k}, D\right)$.

We can prove that $i(T, D)$ is defined uniquely and independent of $T_{k}$. In fact, suppose that $W_{i}: \bar{D} \rightarrow X$ is a $K_{i}$-set-contraction mapping $\left(0<K_{i}<1\right)$ with

$$
\left\|W_{i} x-T x\right\|<\delta \quad x \in \partial D, i=1,2 .
$$

We make a homotopic mapping on $\bar{D}$ as follows

$$
H(t, x)=t W_{1} x+(1-t) W_{2} x \quad x \in \bar{D}, t \in[0,1] .
$$

$H_{t}: \bar{D} \rightarrow X$ is a $\lambda$-set-contraction mapping, where $\lambda=\max \left\{k_{1}, k_{2}\right\}$. For every $x \in \partial D$

$$
\begin{aligned}
\|x-H(t, x)\| & =\left\|x-t W_{1} x-(1-t) W_{2} x\right\| \\
& \geq\|x-T x\|-t\left\|T x-W_{1} x\right\|-(1-t)\left\|T x-W_{2} x\right\| \\
& >\delta-t \delta-(1-t) \delta=0 .
\end{aligned}
$$

Received by the editors September 26, 1984 and, in revised form, August 27, 1987 and December $29,1987$.

1980 Mathematics Subject Classification (1985 Revision). Primary 47H07; Secondary 47H10. 
By Proposition 2.1(3) of [3], $i(H(t, x), D)=$ const. Then

$$
i(H(1, x), D)=i(H(0, x), D),
$$

i.e., $i\left(W_{1}, D\right)=i\left(W_{2}, D\right)$. This equality shows that $i(T, D)$ is independent of $T_{k}$. $i(T, D)$ satisfies three axioms of the fixed-point index:

THEOREM 1. (I) Let $T x=x_{0} \forall x \in \bar{D}$;

$$
i(T, D)= \begin{cases}1, & x_{0} \in D \\ 0, & x_{0} \notin D .\end{cases}
$$

(II) Suppose that $D, D_{1}$, and $D_{2}$ are open subsets of $X, D_{1} \cup D_{2} \subset D$ and $D_{1} \cap$ $D_{2}=\varnothing, T$ has no fixed-point on $\bar{D} \backslash\left(D_{1} \cup D_{2}\right)$. Then $i(T, D)=i\left(T, D_{1}\right)+i\left(T, D_{2}\right)$.

(III) Suppose that the mapping $H:[0,1] \times \bar{D} \rightarrow X$ is continuous and $\theta \notin$ $(I-H(t, x))([0,1] \times \partial D)$. Suppose that for every $t \in[0,1], H(t, \cdot): \bar{D} \rightarrow X$ is a 1-set-contraction mapping. Finally suppose that $H(\cdot, x):[0,1] \rightarrow X$ is uniformly continuous with respect to $x \in \bar{D}$. Then $i(H(1, x), D)=i(H(0, x), D)$ and $i(H(t, x), D)$ is independent of $t$.

PROOF. (I) Since $T$ is a constant mapping, $T$ must be a 0 -set-contraction mapping. Hence

$$
i(T, D)= \begin{cases}1, & x_{0} \in D, \\ 0, & x_{0} \notin D .\end{cases}
$$

(II) As discussion in Definition 1, we set

$$
\delta=\inf \left\{\|x-T x\| \mid x \in \bar{D} \backslash\left(D_{1} \cup D_{2}\right)\right\} .
$$

Suppose that $W: \bar{D} \rightarrow X$ is a strict-set-contraction mapping such that

$$
\|T x-W x\|<\delta, \quad x \in \bar{D} \backslash\left(D_{1} \cup D_{2}\right) .
$$

By Definition 1,

$$
i(T, D)=i(W, D) \quad \text { and } \quad\left(T, D_{j}\right)=i\left(W, D_{j}\right) \quad(j=1,2) .
$$

$W$ has no fixed point on $\bar{D} \backslash\left(D_{1} \cup D_{2}\right)$.

In fact, if there is $x_{0} \in \bar{D} \backslash\left(D_{1} \cup D_{2}\right)$ such that $x_{0}=W x_{0}$, then

$$
\delta \leq\left\|x_{0}-T x_{0}\right\|=\left\|x_{0}-W x_{0}+W x_{0}-T x_{0}\right\|=\left\|W x_{0}-T x_{0}\right\|,
$$

which contradicts (3).

By Proposition 2.1 of $[3], i(W, D)=i\left(W, D_{1}\right)+i\left(W, D_{2}\right)=i\left(T, D_{1}\right)+i\left(T, D_{2}\right)$; then $i(T, D)=i\left(T, D_{1}\right)+i\left(T, D_{2}\right)$.

(III) By the definition of $H(t, x)$, there are $\delta>0$ and $M>0$ such that

$$
\|x-H(t, x)\| \geq \delta>0 \quad t \in[0,1], x \in \partial D,
$$

and $\|H(t, x)\|<M, t \in[0,1], x \in \bar{D}$.

Set $k \in(1-\delta / 2 M, 1)$,

$$
\begin{gathered}
G(t, x)=k H(t, x):[0,1] \times \bar{D} \rightarrow X \\
\|H(t, x)-G(t, x)\| \leq(1-k) M<\delta / 2, \quad t \in[0,1], x \in \bar{D} ;
\end{gathered}
$$

then

$$
\|G(t, x)-x\| \geq\|x-H(t, x)\|-\|H(t, x)-G(t, x)\|>\delta-\delta / 2>0 .
$$


For every $t \in[0,1]$, obviously $G(t, x): \bar{D} \rightarrow X$ is a $k$-set-contraction and $G(\cdot, x)$ : $[0,1] \rightarrow X$ is uniformly continuous with respect to $x \in \bar{D}$.

By Proposition 2.1(3) of [3],

$$
i(G(t, x), D)=\text { const } . \quad(t \in[0,1]) .
$$

According to Definition 1

$$
i(H(t, x), D)=i(G(t, x), D),
$$

then

$$
i(H(1, x), x)=i(H(0, x), D) \text { Q.E.D. }
$$

DEFINITION 2. $T$ is called a semiclosed 1-set-contraction mapping, if $T$ is 1-setcontraction and $I-T$ is closed.

Let us state a definition of semicompact mapping [6].

$T: E \rightarrow E$ is said to be semicompact if for each bounded sequence $\left\{x_{n}\right\}$ in $E$ such that $x_{n}-T\left(x_{n}\right) \rightarrow y$ for some $y$ in $E$, there exists a convergent subsequence.

Obviously, semicompact 1-set-contraction mapping $\rightleftharpoons$ semiclosed 1-setcontraction mapping.

THEOREM 2 (SOLVABILITY). Let $E$ be a real Banach space, $X$ be a closed convex subset of $E, D$ be a bounded open set with respect to $X$.

Suppose that $T: \bar{D} \rightarrow X$ is semiclosed 1-set-contraction mapping, $\theta \notin(I-T) \partial D$, $i(T, D) \neq 0$. Then $T$ has a fixed point in $D$.

PROOF. Suppose that $k_{n} \in(0,1)$ and $k_{n} \rightarrow 1$. We set $W_{n}=k_{n} T, W_{n}: \bar{D} \rightarrow X$ is $k_{n}$-set-contraction. Since $\operatorname{Sup}_{x \in \bar{D}}\|T x\|<+\infty$,

$$
\left\|T x-W_{n} x\right\|=\left\|T x-k_{n} T x\right\|=\left(1-k_{n}\right)\|T x\| \rightarrow 0 \quad(n \rightarrow \infty),
$$

then there is $N$, such that for every $n>N$

$$
\left\|T x-W_{n} x\right\|<\delta \quad \text { where } 0<\delta<\inf _{x \in \partial D}\|x-T x\| .
$$

By the definition and our assumptions, $i(T, D)=i\left(W_{n}, D\right) \neq 0$. By Proposition 2.1(1) of [3], $W_{n}$ has a fixed point $x_{n} \in D$, i.e., $x_{n}=W_{n} x_{n}$. Hence for every $n>N$

$$
\left\|x_{n}-T x_{n}\right\|=\left\|x_{n}-W_{n} x_{n}-T x_{n}+W_{n} x_{n}\right\|=\left\|W_{n} x_{n}-T x_{n}\right\| \rightarrow 0 \quad(n \rightarrow \infty) \text {. }
$$

Then $x_{n}-T x_{n} \rightarrow \theta(n \rightarrow \infty)$.

Since $I-T$ is closed, so $\theta \in(I-T) \bar{D}$, there is $x_{0} \in \bar{D}$ such that $x_{0}=T x_{0}$. Because $\theta \notin(I-T) \partial D$, there exists $x_{0} \in D$ such that $x_{0}=T x_{0}$. Q.E.D.

THEOREM 3. Let $E$ be a real Banach space, $X$ be a closed convex set in $E, D$ be a bounded open set in $X$. Suppose that $T: \bar{D} \rightarrow X$ is a semiclosed 1-set-contraction mapping, and that $x_{0} \in D$ is such that

$$
x \neq t T x+(1-t) x_{0}, \quad x \in \partial D, t \in(0,1] .
$$

Then $T$ has a fixed point in $\bar{D}$.

PROOF. If $T$ has a fixed point on $\partial D$, the conclusion of this theorem obviously holds. 
If $T$ has no fixed point on $\partial D$, then $i(T, D)$ has meaning and

$$
x \neq t T x+(1-t) x_{0} \quad x \in \partial D, t \in[0,1] .
$$

Set

$$
H(t, x)=t T x+(1-t) x_{0} .
$$

We know easily that $H(t, x)$ satisfies the conditions of Theorem 1 (III), so that

$$
i(T, D)=i\left(x_{0}, D\right)=1 \text {. }
$$

By Theorem 2, $T$ has a fixed point in $D$. Q.E.D.

If $T(\partial D) \subset D, x_{0} \in D$, for arbitrary $x \in \partial D, 0<t \leq 1$, we have that

$$
t T x+(1-t) x_{0} \neq x, \quad x \in \partial D .
$$

Then the condition (a) is satisfied. We have the following consequences:

COROllaRY 1. If $T(\partial D) \subset D$, then $T$ has a fixed point in $D$, where $D$ is convex.

Corollary 2. If $x_{0}=\theta \in D$ and $x \neq T x, x \in \partial D, t \in(0,1]$, then $i(T, D)=1$, and $T$ has a fixed point in $D$.

THEOREM 4. Let $E$ be a real Banach space, $X$ be a wedge in $E$ (Amann [5]), and $D$ be a bounded open set in $X$. Suppose that $T: \bar{D} \rightarrow X$ is a semiclosed 1-set-contraction mapping and $x_{0} \in X, x_{0} \neq \theta$ such that

$$
x \neq T x+\lambda x_{0}, \quad \lambda \geq 0, x \in \partial D,
$$

then $i(T, D)=0$.

ProOF. Since $\bar{D}$ is bounded, there exists $\lambda_{0}>0$ such that $\forall \lambda \geq \lambda_{0}, \lambda x_{0} \notin \bar{D}$. We set

$$
H_{\lambda}(t, x)=(1-t) T x+t \lambda x_{0}, \quad \lambda \geq \lambda_{0}, t \in[0,1], x \in \bar{D} .
$$

For arbitrary $t \in[0,1]$ and $\lambda \geq \lambda_{0}, H_{\lambda}(t, \cdot): \bar{D} \rightarrow X$ is a 1-set-contraction mapping.

We can prove that $H_{\lambda}(t, x) \neq x$, where $x \in \partial D, t \in[0,1], \lambda \geq \lambda_{1}$ for some $\lambda_{1} \geq \lambda_{0}$, in fact, if the present inequality does not hold, then there are $\lambda_{n} \rightarrow \infty$ and corresponding $x_{n} \in \partial D$ and $t_{n} \in[0,1]$ such that

$$
x_{n}=\left(1-t_{n}\right) T x_{n}+t_{n} \lambda_{n} x_{0} \text {. }
$$

Since the set $\left\{x_{n}-\left(1-t_{n}\right) T x_{n}\right\}$ is bounded (and hence $\left\{t_{n} \lambda_{n}\right\}$ is bounded), we may suppose that $\lambda_{n} t_{n} \rightarrow l_{0}<+\infty$; hence $t_{n} \rightarrow 0(n \rightarrow \infty)$, and

$$
x_{n}-T x_{n}=\left[x_{n}-\left(1-t_{n}\right) T x_{n}\right]-t_{n} T x_{n} \rightarrow l_{0} x_{0} \text {. }
$$

Because $I-T$ is closed, $l_{0} x_{0} \in(I-T) \partial D$. Then there is $x^{*} \in \partial D$ such that

$$
(I-T) x^{*}=l_{0} x_{0}
$$

which contradicts our assumptions. By Theorem 1 and $\lambda x_{0} \notin \bar{D}\left(\lambda \geq \lambda_{0}\right)$, we obtain

$$
i(T, D)=i\left(\lambda x_{0}, D\right)=0 . \quad \text { Q.E.D. }
$$


THEOREM 5. Let $E$ be a real Banach space, $X$ be a wedge in $E, D$ and $\Omega$ be bounded open sets in $X, \theta \in \Omega \subset \bar{\Omega} \subset D$. Suppose that $T: \bar{D} \rightarrow X$ is a semiclosed 1-set-contraction mapping, and $x_{2} \in X, x_{1} \in D$ such that

(i) $x \neq t T x+(1-t) x_{1}, x \in \partial D, t \in(0,1]$.

(ii) $x \neq T x+t x_{2}, x \in \partial \Omega, t \geq 0$.

Then there exists $x_{0} \in \bar{D} \backslash \Omega$ such that $x_{0}=T x_{0}$.

PROOF. If there exists $x_{0} \in \partial D$ such that $x_{0}=T x_{0}$, this theorem is proved.

If for $x \in \partial D, x \neq T x$, then from the proof of Theorem 3 and by Theorem 4 and Theorem 1(II) we obtain

$$
i(T, D \backslash \bar{\Omega})=i(T, D)-i(T, \Omega)=1 .
$$

Therefore there exists $x_{0} \in D \backslash \bar{\Omega}$ such that $x_{0}=T x_{0}$. Q.E.D.

COROLlaRY. Let $P$ be a cone in real Banach space $X$, and $T: \bar{P}_{r} \rightarrow P$ a semiclosed 1-set-contraction mapping, then there exist $r_{1}, r_{2}: 0<r_{2}<r_{1}<r$ such that

(i) For all $x \in \partial P_{r_{1}}$ and $\lambda \geq 1, T x \neq \lambda x$.

(ii) There exists $x_{0} \in P\left(x_{0} \neq \theta\right)$ such that for all $x \in \partial P_{r_{2}}$ and $\lambda \geq 0, x-T x \neq$ $\lambda x_{0}$, where $P_{r}=\{x \in P\|\| x \|<r\}, \partial P_{r}=\{x \in P \mid\|x\|=r\}$.

Then $T$ has a fixed point $x^{*} \in P_{r_{1}, r_{2}}$, i.e., $r_{2}<\left\|x^{*}\right\|<r_{1}$.

THEOREM 6. Let $T: P_{r} \rightarrow P$ be a semiclosed 1-set-contraction mapping and $r_{1}>r_{2}>0\left(r_{1}<r\right)$ such that the following holds:

(i) $x \in \partial P_{r_{1}} \Rightarrow T x \nsupseteq x ; x \in \partial P_{r_{2}} \Rightarrow x \nsucceq T x$, or

(ii) $x \in \partial P_{r_{2}} \Rightarrow T x \nsucceq x ; x \in \partial P_{r_{1}} \Rightarrow x \nsupseteq T x$.

Then there exists a fixed point $x^{*} \in P_{r_{1}, r_{2}}$, i.e., $r_{2}<\left\|x^{*}\right\|<r_{1}$.

PROOF. By the corollary of Theorem 5 we obtain this theorem.

LEMMA. Let $P$ be a cone of real Banach space $E$ and the norm be monotonically increasing with respect to $P$. Suppose that $A: \bar{P}_{r, R} \rightarrow P$ is a K-set-contraction mapping $(0<K<1)$ which satisfies the following conditions:

$\left(\mathrm{H}_{1}\right) x \in \partial P_{r} \Rightarrow\|A x\| \leq\|x\| ; x \in \partial P_{R} \Rightarrow\|A x\| \geq\|x\|$, or

$\left(\mathrm{H}_{2}\right) x \in \partial P_{R} \Rightarrow\|A x\| \leq\|x\| ; x \in \partial P_{r} \Rightarrow\|A x\| \geq\|x\|$.

Then $A$ has a fixed point in $\bar{P}_{r, R}$, where $P_{r, R}=P_{R} \backslash \bar{P}_{r}$.

PROOF. We prove only that this theorem holds under $\left(\mathrm{H}_{1}\right)$. Mapping $A$ is a strict-set-contraction. Set $s=\frac{1}{2}(r+R)$.

We set operator $A_{n}$ as follows:

$$
A_{n} x= \begin{cases}\left(1+\frac{\|x\|-s}{n(R-s)}\right) A x, & x \in P, s \leq\|x\| \leq R, \\ \left(1-\frac{s-\|x\|}{n(s-r)}\right) A x, & x \in P, r \leq\|x\|<s .\end{cases}
$$

$A_{n}$ is a continuous and bounded operator.

We discuss operator

$$
B_{n} x=\frac{\|x\|}{n k} A x, \quad x \in \bar{P}_{r, R}, k=\min \{R-s, r-s\}=\frac{1}{2}(R-r) .
$$


Let $\Omega$ be an arbitrary open subset in $\bar{P}_{r, R}$. By definition and property of the measure $\alpha(\Omega)$ of noncompactness of $\Omega$,

$$
\begin{aligned}
& \alpha(\Omega)=\inf \left\{d \mid s_{i} \subset \Omega, \bigcup_{n=1}^{N} s_{i}=\Omega, \operatorname{diam} s_{i}<d\right\} \\
& \alpha(\Omega) \leq \alpha\left\{\bar{P}_{r, R}\right\}, \quad B_{n}\left(s_{i}\right) \subset B_{n}(\Omega), \quad \bigcup_{i=1}^{N} B_{n}\left(s_{i}\right)=B_{n}(\Omega), \\
& \operatorname{diam} B_{n}\left(s_{i}\right) \leq \frac{2 R}{n k} \operatorname{diam} A\left(s_{i}\right), \\
& \alpha\left(B_{n}(\Omega)\right) \leq \frac{2 R}{n k} \alpha(A(\Omega)) \leq \frac{2 R}{n k} K \alpha(\Omega) .
\end{aligned}
$$

Then $B_{n}$ is a $(2 R / n k) K$-set-contraction mapping. As $n \rightarrow \infty,(2 R / n k) K \rightarrow 0$, i.e., when $n$ is large enough, $B_{n}$ is a strict-set-contraction mapping on $\bar{P}_{r, R}$.

In addition, we set operators:

$$
\begin{array}{ll}
A_{n}^{(1)} x=\left(1+\frac{\|x\|-s}{n(R-s)}\right) A x, & x \in P, s \leq\|x\| \leq R, \\
A_{n}^{(2)} x=\left(1-\frac{s-\|x\|}{n(s-r)}\right) A x, & x \in P, r \leq\|x\|<s .
\end{array}
$$

Obviously, $A_{n}^{(1)}$ and $A_{n}^{(2)}$ are $\tilde{k}$-set-contraction mappings, where $\tilde{k} \leq(1+(2 R+$ $s / n k)) K$. When $n$ is large enough, $0<\tilde{k}<1$. Hence $A_{n}^{(1)}$ and $A_{n}^{(2)}$ are strict-setcontraction mappings for $n$ large enough. Set

$$
P_{1}=\{x \in P \mid s \leq\|x\| \leq R\}, P_{2}=\{x \in P \mid r \leq\|x\|<s\}, \quad \bar{P}_{r, R}=P_{1} \cup P_{2} .
$$

Hence, $\Omega=\left(\Omega \cap P_{1}\right) \cup\left(\Omega \cap P_{2}\right)$,

$$
\begin{aligned}
& A_{n}(\Omega)=\left[A_{n}\left(\Omega \cap P_{1}\right)\right] \cup\left[A_{n}\left(\Omega \cap P_{2}\right)\right], \\
&=\left[A_{n}^{(1)}\left(\Omega \cap P_{1}\right)\right] \cup\left[A_{n}^{(2)}\left(\Omega \cap P_{2}\right)\right], \\
& \alpha\left(A_{n}(\Omega)=\right. \alpha\left\{\left[A_{n}^{(1)}\left(\Omega \cap P_{1}\right)\right] \cup\left[A_{n}^{(2)}\left(\Omega \cap P_{2}\right)\right]\right\}, \\
&= \operatorname{Max}\left\{\alpha\left[A_{n}^{(1)}\left(\Omega \cap P_{1}\right)\right], \alpha\left[A_{n}^{(2)}(\Omega \cap P)\right]\right\}, \\
& \leq \tilde{k} \alpha(\Omega), \quad 0<\tilde{k}<1 .
\end{aligned}
$$

Then $A_{n}$ is a strict-set-contraction mapping and when $n$ is large enough, $A_{n}$ satisfies the conditions of Theorem 6 .

In fact, if there is $x_{0} \in \partial P_{r}$ such that $A_{n} x_{0} \geq x_{0}$, then the norm $\|x\|$ is monotonically increasing and by condition $\left(\mathrm{H}_{1}\right)$,

$$
r=\left\|x_{0}\right\| \leq\left\|A_{n} x_{0}\right\|=(1-1 / n)\left\|A x_{0}\right\| \leq(1-1 / n)\left\|x_{0}\right\|=(1-1 / n) r<r .
$$

This is a contradiction. Then $x \in \partial P_{r}, A_{n} x \nsucceq x$.

If there is $x_{1} \in \partial P_{R}$ such that $A_{n} x_{1} \leq x_{1}$, we obtain the following:

$$
R=\left\|x_{1}\right\| \geq\left\|A_{n} x_{1}\right\|=(1+1 / n)\left\|A x_{1}\right\| \geq(1+1 / n)\left\|x_{1}\right\|=(1+1 / n) R>R .
$$

This is also a contradiction. Then $x \in \partial P_{R}, A_{n} x \not x$. Hence $A_{n}$ satisfies the conditions of Theorem 6 . 
There is $x^{*} \in \bar{P}_{r, R}$ such that $A_{n} x^{*}=x^{*}$. Without loss of generality, suppose that $P_{1}$ includes subsequence $\left\{x_{n_{k}}^{*}\right\}$ of $\left\{x_{n}^{*}\right\}$,

$$
x_{n_{k}}^{*}=A_{n_{k}} x_{n_{k}}^{*}=\left(1+\frac{\left\|x_{n_{k}}^{*}\right\|-s}{n_{k}(R-s)}\right) A x_{n_{k}}^{*} .
$$

Since $A$ is a strict-set-contraction mapping, the set $\left\{\left\|A x_{n_{k}}^{*}\right\|\right\}$ must be bounded:

$$
\left\|x_{n_{k}}^{*}-A x_{n_{k}}^{*}\right\|=\frac{\left\|x_{n_{k}}^{*}\right\|-s}{n_{k}(R-s)}\left\|A x_{n_{k}}^{*}\right\|<\frac{1}{n_{k}}\left\|A x_{n_{k}}^{*}\right\| \rightarrow 0 \quad\left(n_{k} \rightarrow \infty\right),
$$

i.e., $x_{n_{k}}^{*}-A x_{n_{k}}^{*} \rightarrow \theta\left(n_{k} \rightarrow \infty\right)$.

Since a strict-set-contraction mapping is a semicompact 1-set-contraction, then there exists a convergent subsequence of $\left\{x_{n_{k}}^{*}\right\}$, which we write down also as $\left\{x_{n_{k}}^{*}\right\}$, and $x_{n_{k}}^{*} \rightarrow x^{*}\left(n_{k} \rightarrow \infty\right)$. Since $\bar{P}_{r, R}$ is closed, $x^{*} \in \bar{P}_{r, R}$. By (4), as $n_{k} \rightarrow \infty$, we imply that $A x^{*}=x^{*}$. This proves that $A$ has a fixed point $x^{*} \in \bar{P}_{r, R}$ under $\left(\mathrm{H}_{1}\right)$. Analogously, we can prove that $A$ has a fixed point $x^{*} \in \bar{P}_{r, R}$ under $\left(\mathrm{H}_{2}\right)$.

THEOREM 7. Let $P$ be a cone of a real Banach space $E$ and let the norm be monotone with respect to $P$. Suppose that $A: \bar{P}_{r, R} \rightarrow P$ is a semiclosed 1-setcontraction mapping and there is $\delta>0$ such that

(i) $x \in \partial P_{r} \Rightarrow\|A x\| \leq\|x\| ; x \in \partial P_{R} \Rightarrow\|A x\| \geq(1+\delta)\|x\|$, or

(ii) $x \in \partial P_{R} \Rightarrow\|A x\| \leq\|x\| ; x \in \partial P_{r} \Rightarrow\|A x\| \geq(1+\delta)\|x\|$.

Then $A$ has a fixed point in $\bar{P}_{r, R}$.

ProOF. (i) Since $A: \bar{P}_{r, R} \rightarrow P$ is a semiclosed 1-set-contraction mapping, then for an arbitrary open subset $\Omega \subset \bar{P}_{r, R}$,

$$
\alpha(A(\Omega)) \leq \alpha(\Omega)
$$

We construct operator $A_{n}$ as follows:

$$
A_{n} x=\lambda_{n} A, \lambda_{n}=\frac{n-1}{n} .
$$

Hence $\alpha\left(A_{n}(\Omega)\right)=\alpha\left(\lambda_{n}(A(\Omega))=\lambda_{n} \alpha(A(\Omega)) \leq \lambda_{n} \alpha(\Omega)\right.$. Hence $A_{n}$ is a strict-setcontraction mapping.

If the condition (i) holds, when $n$ is large enough that $1>\lambda_{n}>1 /(1+\delta)$, then

$$
\begin{array}{ll}
\text { for } x \in \partial P_{r} & \left\|A_{n} x\right\|=\lambda_{n}\|A x\|<\|A x\| \leq\|x\| ; \\
\text { for } x \in \partial P_{R} & \left\|A_{n} x\right\|=\left\|\lambda_{n} A x\right\|=\lambda_{n}\|A x\|>\frac{1}{1+\delta}\|A x\| \geq\|x\| .
\end{array}
$$

$A_{n}$ satisfies the conditions of the lemma, hence there exists $x_{n} \in \bar{P}_{r, R}$, such that

$$
\begin{aligned}
& A_{n} x_{n}=x_{n}, \quad \text { i.e., } \lambda_{n} A x_{n}=x_{n}, \\
& x_{n}-A x_{n}=x_{n}-\lambda_{n} A x_{n}+\lambda_{n} A x_{n}-A x_{n}=\left(\lambda_{n}-1\right) A x_{n} \rightarrow \theta \quad(n \rightarrow \infty) .
\end{aligned}
$$

Because $(I-A)$ is closed and $\bar{P}_{r, R}$ is a closed set, there is $x_{0} \in \bar{P}_{r, R}$ such that $x_{0}=A x_{0}$.

Analogously we can prove that $A$ has a fixed point $x^{*} \in \bar{P}_{r, R}$ under condition (ii). Q.E.D. 
THEOREM 8. Let $E$ be a real Banach space and $P$ be a normal cone of $E$. Suppose that $T:[0, y] \rightarrow E$ is a monotonically increasing semiclosed 1-set-contraction mapping and $T(0) \geq 0, T(y) \leq y$. Then $T$ has a fixed point in $[0, y]$.

PROOF. We set $T_{m} x=t_{m} T x$, where $m$ is an arbitrary natural number and $t_{m}=(m-1) / m$. Obviously, $T_{m}:[0, y] \rightarrow E$ is a monotonically increasing $t_{m}$-setcontraction mapping $\left(0<t_{m}<1\right)$. We have

$$
T_{m}(0)=t_{m} T(0) \geq t_{m} \cdot 0 \geq 0 ; \quad T_{m}(y)=t_{m} T(y) \leq t_{m} y \leq y ;
$$

hence $T_{m}$ satisfies the conditions of Theorem 3 of [3], it has a minimal fixed point $\tilde{x}_{m}$ and

$$
\begin{aligned}
& \tilde{x}_{m}=\lim _{n \rightarrow \infty} T_{m}^{(n)}(0), \\
& \tilde{x}_{m}-T\left(\tilde{x}_{m}\right)=T_{m}\left(\tilde{x}_{m}\right)-T\left(\tilde{x}_{m}\right)=\left(t_{m}-1\right) T\left(\tilde{x}_{m}\right) \rightarrow \theta \quad(m \rightarrow \infty) .
\end{aligned}
$$

Since $(I-T)$ is closed, $\theta \in(I-T)[0, y]$. Then there is $\bar{x} \in[0, y]$ such that $\bar{x}-T \bar{x}=\theta$, i.e., $\bar{x}=T \bar{x}$. Q.E.D.

The author is grateful to the referee for a number of useful suggestions.

\section{REFERENCES}

1a. W. V. Petryshyn, Trans. Amer. Math. Soc. 182 (1973), 323-352.

1b. _ Bull. Amer. Math. Soc. 78 (1972), 225-229.

2. D. D. Nussbaum, Ann. Math. Pura. Appl. 89 (1971), 217-258.

3. H. Amann, J. Funct. Anal. 11 (1972), 346-384.

4. N. P. Cac and J. A. Gatica, J. Math. Anal. Appl. 71 (1979), 547-557.

5. H. Amann, SIAM Rev. (1976), 632.

6. W. V. Petryshyn, J. Math. Anal. and Appl. 39 (1972), 717-741.

7. Zhang Qing Yong, Chinese Ann. of Math. (2) 5 (1984), 91-98.

Department of Mathematics, Jiangxi Normal University, Nanchang, PEOPLE'S REBUPLIC OF CHINA

Department of MAThematics, Fordham University, BronX, NeW York, 10458 